\title{
Produtividade simulada de tubérculos de batata em cenários de mudanças climáticas
}

\author{
Joelma Dutra Fagundes(1), Nereu Augusto Streck(1), Dilson Antônio Bisognin ${ }^{(1)}$, \\ Ana Paula Schwantes ${ }^{(1)}$ e Cleber Maus Alberto ${ }^{(2)}$
}

\begin{abstract}
(1)Universidade Federal de Santa Maria, Departamento de Fitotecnia, Avenida Roraima, no 1.000, Bairro Camobi, CEP 97105-900 Santa Maria, RS. E-mail: jdfagundes@hotmail.com, nstreck2@yahoo.com.br, dbisognin@gmail.com, apschwantes@hotmail.com (2)Universidade Federal do Pampa, Rua Luiz Joaquim de Sá Britto, s/no, CEP 97650-000 Itaqui, RS. E-mail: cleberalberto@unipampa.edu.br
\end{abstract}

\begin{abstract}
Resumo - O objetivo deste trabalho foi simular a produtividade de tubérculos de batata (Solanum tuberosum) em diferentes cenários climáticos de aumento da concentração de dióxido de carbono $\left[\mathrm{CO}_{2}\right]$ e da temperatura do ar, considerando aumentos simétricos e assimétricos nas temperaturas mínimas e máximas diárias do ar. Utilizou-se o modelo de Spitters para simular a produtividade de tubérculos da cultivar Asterix, em duas épocas de cultivo (primavera e outono), recomendadas para a região de Santa Maria, RS. Em cada época, cinco datas de plantio foram avaliadas, em cenários climáticos de cem anos sem aumentos na $\left[\mathrm{CO}_{2}\right]$ e na temperatura (cenário atual), e em cenários com o dobro da $\left[\mathrm{CO}_{2}\right]$ atual e aumentos de $0,1,2,3,4,5$ e $6^{\circ} \mathrm{C}$ na temperatura. No cultivo de primavera, o aumento simétrico de $4^{\circ} \mathrm{C}$, nas temperaturas mínimas e máximas diárias do ar, e assimétrico de $5^{\circ} \mathrm{C}$ é suficiente para anular o efeito benéfico na produtividade do aumento da $\left[\mathrm{CO}_{2}\right]$. No cultivo de outono, o aumento da temperatura, praticamente, não afeta a produtividade de tubérculos de batata. A antecipação da data de plantio, no cultivo de primavera, e o atraso, no de outono, diminuem o impacto negativo do aumento da temperatura do ar sobre a produtividade de tubérculos de batata.
\end{abstract}

Termos para indexação: Solanum tuberosum, aquecimento global, concentração de dióxido de carbono, fenologia, simulação de risco climático.

\section{Potato tuber simulated yield in climate change scenarios}

Abstract - The objective of this study was to simulate potato (Solanum tuberosum) tuber yield in different climate change scenarios of increased carbon dioxide concentration $\left[\mathrm{CO}_{2}\right]$ and air temperature, considering symmetric and asymmetric increases in minimum and maximum daily air temperatures. Spitters model was used to simulate Asterix cultivar tuber yield considering two growing seasons (spring and fall) recommended for Santa Maria, state of Rio Grande do Sul, Brazil. In each growing season, five planting dates were evaluated in climate scenarios of a hundred years with no increase in $\left[\mathrm{CO}_{2}\right]$ and temperature (current scenario), and in scenarios with doubling $\left[\mathrm{CO}_{2}\right]$ and temperature increases of $0,1,2,3,4,5$ and $6^{\circ} \mathrm{C}$. A symmetric increase of $4^{\circ} \mathrm{C}$ and an asymmetric increase of $5^{\circ} \mathrm{C}$ in air temperature offset the yield beneficial effect of increasing $\left[\mathrm{CO}_{2}\right]$ during spring, whereas increase in air temperature does not affect potato tuber yield during fall. Anticipating planting date in spring and delaying it in fall decrease the negative impact of the increasing air temperature on potato tuber yield.

Index terms: Solanum tuberosum, global warming, carbon dioxide concentration, phenology, climate risk simulation.

\section{Introdução}

Estudos numéricos com modelos de circulação geral da atmosfera (General Circulation Models - GCM) indicam que é bastante provável que se verifiquem aumentos de 1,1 a $6,4^{\circ} \mathrm{C}$ na temperatura média do ar até o final deste século, em vários locais do planeta (Intergovernmental Panel on Climate
Change, 2007), até mesmo no Brasil (Siqueira et al., 2001; Assad et al., 2004; Intergovernmental Panel on Climate Change, 2007).

Esse aumento na temperatura do ar deve ocorrer em razão do aumento na concentração de dióxido de carbono $\left[\mathrm{CO}_{2}\right]$ atmosférico, que deverá duplicar em algum momento deste século (Streck, 2005; Intergovernmental Panel on Climate Change, 2007). 
Registros indicam que, durante o século $\mathrm{XX}$, houve aumento na temperatura média da superfície global de $0,6 \pm 0,2^{\circ} \mathrm{C}$, sendo que a década de 1990 foi considerada a mais quente do último milênio (Kerr, 2005; Intergovernmental Panel on Climate Change, 2007). Segundo Karl et al. (1991), o aquecimento global pode se caracterizar por um aumento assimétrico na temperatura mínima e máxima do ar, com maior aumento na temperatura mínima do que na temperatura máxima diária.

A concentração de $\mathrm{CO}_{2}$ atmosférico tem influência no desenvolvimento, crescimento e produtividade dos cultivos agrícolas. Se a concentração desse gás aumentar, a taxa de crescimento das plantas poderá aumentar em razão de o $\mathrm{CO}_{2}$ ser o substrato primário para a fotossíntese (Taiz \& Zeiger, 2004). A expectativa é de que plantas com metabolismo $\mathrm{C}_{3}$, em razão de sua morfofisiologia, sejam mais beneficiadas do que plantas $\mathrm{C}_{4}$ (Tubiello et al., 2000; Siqueira et al., 2001; Streck, 2005). Streck (2005) relata que, se a $\left[\mathrm{CO}_{2}\right]$ dobrar em relação à atual (380 ppmv), deverá ocorrer um aumento médio de $30 \%$ na produtividade de culturas $\mathrm{C}_{3}$ e de $10 \%$ na produtividade de culturas $\mathrm{C}_{4}$. No entanto, se o aumento da concentração de $\mathrm{CO}_{2}$ for acompanhado pelo aumento da temperatura do ar, o maior crescimento e produtividade das culturas poderá ser anulado, em razão do encurtamento do ciclo (Siqueira et al., 2001) e do aumento da respiração (Taiz \& Zeiger, 2004).

Os agroecossistemas são suscetíveis a mudanças climáticas (Intergovernmental Panel on Climate Change, 2007) e, por isso, estudos do impacto das mudanças climáticas sobre os agroecossistemas são importantes para a tomada de decisão na agricultura. Porém, é difícil que esses estudos sejam realizados experimentalmente, pela limitação de recursos humanos e financeiros (Andresen et al., 2001). Uma ferramenta auxiliar aos experimentos com estudos do impacto das mudanças climáticas sobre as culturas agrícolas são os modelos matemáticos, os quais se constituem em uma simplificação da realidade dos agroecossistemas (Streck \& Alberto, 2006a).

No Brasil, estudos do impacto das mudanças climáticas sobre a agricultura, com uso de modelos matemáticos, já foram realizados com várias culturas em diversas regiões brasileiras (Siqueira et al., 2000, 2001; Assad et al., 2004; Gondim et al., 2008), inclusive em Santa Maria (Streck \& Alberto, 2006a).
No entanto, no que se refere à cultura da batata, que ocupa o quarto lugar em volume de produção mundial de alimentos, sendo superada apenas pelo trigo, arroz e milho (Nyende et al., 2005), o único trabalho em condições brasileiras encontrado na literatura é o de Streck et al. (2006). Contudo, nesse trabalho, avaliou-se a resposta fenológica da batata a cenários de mudanças climáticas.

Assim, resta analisar a resposta da produtividade de tubérculos da batata em condições brasileiras, em cenários futuros de mudanças climáticas. Rosenzweig et al. (1996), nos Estados Unidos, relataram redução na produtividade de tubérculos de batata de 1,4, 3,4 e $18,5 \%$, respectivamente, para aumentos de $1,5,2,5$ e $5,0^{\circ} \mathrm{C}$. Uma das alternativas para evitar o efeito negativo de altas temperaturas sobre a produtividade agrícola seria modificar a data de plantio. A mudança na data de plantio, nesse caso, diminuiria a exposição a altas temperaturas durante o crescimento dos tubérculos e aumentaria a duração da área foliar verde (Hijmans, 2003), o que diminuiria os efeitos negativos das altas temperaturas do ar na produtividade dos tubérculos de batata (Rosenzweig et al., 1996).

O objetivo deste trabalho foi simular, pelo uso do modelo matemático de Spitters (1987), a produtividade de tubérculos de batata em cenários com aumento da concentração de dióxido de carbono e da temperatura do ar, em diferentes datas de plantio, considerando aumentos simétricos e assimétricos da temperatura mínima e máxima diárias do ar.

\section{Material e Métodos}

Este estudo numérico foi realizado com base nos dados meteorológicos de temperatura mínima e temperatura máxima diárias do ar, durante o período de 1969 a 2003, coletados na Estação Meteorológica do Instituto Nacional de Meteorologia (Oitavo Distrito de Meteorologia), localizada no Departamento de Fitotecnia da Universidade Federal de Santa Maria, RS (294ㄴ $\mathrm{S}, 5^{\circ} 43^{\prime} \mathrm{W}$, e altitude de $\left.95 \mathrm{~m}\right)$. O clima da região, segundo a classificação de Köppen, é Cfa subtropical úmido, sem estação seca definida, com verões quentes.

A série de dados de 1969 a 2003 foi utilizada para a criação dos cenários de mudanças climáticas no LARS-WG Weather Generator, que é um modelo estocástico, frequentemente usado em estudos de 
impacto de cenários de mudanças climáticas sobre o desempenho de agroecossistemas (Weiss et al., 2003; Richter \& Semenov, 2005; Streck \& Alberto, 2006a, 2006b), capaz de produzir séries de dados sintéticos com uso de funções semiempíricas de distribuição de probabilidade, que mantém as estatísticas da série original de dados observados (Semenov et al., 1998).

Assim, o modelo LARS-WG gerou uma série de 100 anos de dados, previamente analisados por Streck \& Alberto (2006a, 2006b), sem que tenham havido diferenças entre as estatísticas das séries de dados geradas pelo LARS-WG e a série de dados observados. Dessa forma, os cenários simulados puderam ser utilizados neste estudo.

Com base nas projeções de aumento de temperatura e concentração de $\mathrm{CO}_{2}$ dos modelos de circulação geral da atmosfera (GCM), foram estabelecidos os cenários climáticos de 100 anos, que correspondem a cenários plausíveis dentro de 100 anos, segundo Intergovernamental Panel on Climate Change (2007). Com esse objetivo foram utilizados dados meteorológicos diários de temperatura mínima e máxima do ar, desde o cenário sem aumento de $\mathrm{CO}_{2}$ e temperatura (clima atual) até o cenário com o dobro de $\mathrm{CO}_{2}$ e acréscimo de $6^{\circ} \mathrm{C}$ na temperatura (climas futuros). Foram considerados aumentos simétricos e assimétricos nas temperaturas mínimas e máximas do

Tabela 1. Cenários climáticos de aumento da concentração de $\mathrm{CO}_{2}$ e da temperatura do ar, com simulação de aumentos simétricos e assimétricos da temperatura mínima e máxima diária, obtida a partir do modelo LARS-WG Weather Generator (Semenov et al., 1998).

\begin{tabular}{|c|c|c|c|c|}
\hline $\begin{array}{l}\text { Cenário } \\
\text { climático (1) }\end{array}$ & $\begin{array}{c}\text { Concentração de } \\
\mathrm{CO}_{2} \text { (ppmv) }\end{array}$ & $\begin{array}{l}\text { Temperatura } \\
\text { mínima }\left({ }^{\circ} \mathrm{C}\right)\end{array}$ & $\begin{array}{l}\text { Temperatura } \\
\text { máxima }\left({ }^{\circ} \mathrm{C}\right)\end{array}$ & $\begin{array}{l}\text { Temperatura } \\
\text { média }\left({ }^{\circ} \mathrm{C}\right)\end{array}$ \\
\hline 1 & 380 & $-{ }^{(2)}$ & $-{ }^{(2)}$ & $-{ }^{(2)}$ \\
\hline$\underline{2}$ & 760 & $--^{(2)}$ & $--^{(2)}$ & $--^{(2)}$ \\
\hline & \multicolumn{4}{|c|}{ Aumentos simétricos de temperatura } \\
\hline $3 a$ & 760 & 1,0 & 1,0 & 1,0 \\
\hline $4 a$ & 760 & 2,0 & 2,0 & 2,0 \\
\hline $5 a$ & 760 & 3,0 & 3,0 & 3,0 \\
\hline $6 \mathrm{a}$ & 760 & 4,0 & 4,0 & 4,0 \\
\hline $7 \mathrm{a}$ & 760 & 5,0 & 5,0 & 5,0 \\
\hline \multirow[t]{2}{*}{$8 \mathrm{a}$} & 760 & 6,0 & 6,0 & 6,0 \\
\hline & \multicolumn{4}{|c|}{ Aumentos assimétricos de temperatura } \\
\hline $3 b$ & 760 & 1,2 & 0,8 & 1,0 \\
\hline $4 b$ & 760 & 2,4 & 1,6 & 2,0 \\
\hline $5 b$ & 760 & 3,6 & 2,4 & 3,0 \\
\hline $6 b$ & 760 & 4,8 & 3,2 & 4,0 \\
\hline $7 b$ & 760 & 6,0 & 4,0 & 5,0 \\
\hline $8 \mathrm{~b}$ & 760 & 7,2 & 4,8 & 6,0 \\
\hline
\end{tabular}

${ }^{(1)}$ Cenário 1 , cenário atual sem mudança. Cenário 2, com o dobro da concentração de $\mathrm{CO}_{2}$ do cenário 1, cenários 3 a 8 , aumentos simétricos (a) ou assimétricos (b) de temperaturas máximas e mínimas. ${ }^{(2)}$ Sem alteração. ar (Tabela 1). A densidade diária do fluxo de radiação solar global incidente foi considerada a mesma em todos os cenários.

A fim de simular o impacto das possíveis mudanças climáticas na produtividade de tubérculos de batata, foi utilizado o modelo de simulação da produtividade proposto por Spitters (1987), calibrado e avaliado para a cultivar Asterix nas condições de Santa Maria, $\mathrm{RS}$, em treze datas de plantio durante os anos 2003 e 2004 (Streck et al., 2006). O modelo de Spitters (1987) simula a produtividade de tubérculos considerando que não há efeito de fatores bióticos, como pragas, doenças e plantas daninhas, e nem limitação hídrica ou nutricional sobre a cultura da batata. Assim, o modelo simula a produtividade potencial de tubérculos de batata, que é uma função da disponibilidade de radiação solar e da temperatura do ar. De modo geral, o modelo de Spitters (1987) simula a produtividade de tubérculos de batata a partir da biomassa total e a fração de massa de matéria seca alocada para os tubérculos,

$$
\mathrm{Y}_{\mathrm{t}}=\mathrm{HI}_{\mathrm{t}} \mathrm{x} \mathrm{W}_{\mathrm{t}}
$$

em que: $Y_{t}$ é a produtividade dos tubérculos de batata $\left(\mathrm{kg} \mathrm{ha}^{-1}\right), \mathrm{HI}_{\mathrm{t}}$ é o índice de colheita (fração da massa de matéria seca total alocada para os tubérculos) e $\mathrm{W}_{\mathrm{t}}$ é a biomassa total $\left(\mathrm{kg} \mathrm{ha}^{-1}\right)$.

$\mathrm{O}$ efeito do aumento da concentração de $\mathrm{CO}_{2}$ sobre o desenvolvimento e o crescimento da planta de batata foi considerado no modelo pelo aumento na eficiência de uso da radiação (EUR). Considerou-se um aumento de $26 \%$ na EUR, quando a concentração de $\mathrm{CO}_{2}$ foi o dobro da concentração atual, conforme relatado por Schapendonk et al. (1995) para cultivares de batata de ciclo médio, como é o caso da cultivar Asterix. Assim, no cenário atual (Tabela 1) a EUR foi de 4,72 $\mathrm{g} \mathrm{MJ}^{-1}$, e nos cenários futuros a EUR foi de 5,95 $\mathrm{g} \mathrm{MJ}^{-1}$. Ao modelo de Spitters (1987), foi adicionada uma função de penalização da fotossíntese em resposta à temperatura máxima do ar, pois a EUR de cultivares de batata diminui linearmente com o aumento da temperatura na faixa entre $25^{\circ} \mathrm{C}$ e $35^{\circ} \mathrm{C}$ (Kooman \& Haverkort, 1995).

Assim, para o cenário atual e cenários futuros, a resposta da EUR ao aumento da temperatura máxima diária na faixa de 25 a $35^{\circ} \mathrm{C}$ foi calculada, respectivamente, por EUR $=4,72$ [(-0,1 Tmáx $)+3,5]$; e EUR $=5,95$ [(-0,1 Tmáx $)+3,5]$, em que EUR é a 
eficiência de uso da radiação e Tmáx é a temperatura máxima diária.

Foram avaliadas duas épocas de cultivo em cada ano dos cenários: cultivo de primavera, ou safra, com plantio em 12 de agosto, e cultivo de outono, ou safrinha, com plantio em 12 de fevereiro. Essas datas de plantio estão dentro das épocas recomendadas para os cultivos de primavera e de outono, respectivamente, na região de Santa Maria, RS, que são de agosto a outubro e de janeiro a março (Bisognin et al., 2008). O dia da emergência, considerado como o primeiro dia da simulação, ocorreu 20 dias após o plantio de primavera (1/9) e 10 dias após o plantio de outono (22/2) (Streck et al., 2006). Além dessas duas datas, também foram consideradas duas datas de plantio anteriores e posteriores em cada época de cultivo, de modo que as datas de emergência das plantas ficassem distanciadas sete dias entre si, pois o modelo de Spitters (1987) inicia os cálculos a partir da data de emergência da cultura. Assim, o total das datas de emergência usadas na simulação foram 18/8, 25/8, 1/9, 8/9 e 15/9, para o cultivo de primavera, e $8 / 2,15 / 2,22 / 2,1 / 3$ e $8 / 3$, para o cultivo de outono.

A análise dos dados foi realizada pelo cálculo dos desvios entre os cenários com mudança e o cenário atual (cenário 1 da Tabela 1), para a produtividade de tubérculos de batata $\left(\mathrm{Mg} \mathrm{ha}^{-1}\right)$ em todos os anos, em cada cenário climático, em todas as datas de plantio, nos cultivos de primavera e outono. Nos cenários de mudanças climáticas, o desvio positivo significa aumento de produtividade de tubérculos de batata, enquanto o desvio negativo significa redução (Streck et al., 2006; Lago et al., 2008).

\section{Resultados e Discussão}

Quando simulada no cenário atual, sem mudança em $\left[\mathrm{CO}_{2}\right]$ e na temperatura (cenário 1, Tabela 1), a produtividade de tubérculos de batata para as datas de plantio recomendadas para o cultivo de primavera foi superior à de outono (Figura $1 \mathrm{~A}$ e C), o que está de acordo com os resultados observados em campo (Bisognin et al., 2008), e indica que o modelo de Spitters (1987) reproduz adequadamente o que acontece com a cultura da batata cultivada sob as condições atuais na região de estudo.

No cenário 2, apenas com aumento da $\left[\mathrm{CO}_{2}\right]$, nos cultivos de primavera e outono, foi observado efeito positivo do $\mathrm{CO}_{2}$ sobre a produtividade de tubérculos de batata, quando comparado ao cenário 1 (Figura $1 \mathrm{~A}$ e C). Tal resultado provavelmente ocorreu pela maior taxa de crescimento das plantas proporcionada pela maior EUR, o que fisiologicamente era esperado, já que o $\mathrm{CO}_{2}$ é o substrato primário da fotossíntese (Taiz \& Zeiger, 2004). Quando o aumento na concentração de $\mathrm{CO}_{2}$ foi acompanhado pelo aumento da temperatura do ar, o efeito benéfico do aumento da $\left[\mathrm{CO}_{2}\right]$ nos cenários $3 \mathrm{a}, 3 \mathrm{~b}, 4 \mathrm{a}, 4 \mathrm{~b}$ e $5 \mathrm{~b}$ persistiram no cultivo de primavera, enquanto, em outros cenários desse cultivo, o efeito benéfico do aumento da $\left[\mathrm{CO}_{2}\right]$ foi anulado, uma vez que são observados desvios negativos nos cenários 5a, 6, 7 e 8 (Figura 1 A e B). Os desvios negativos ocorreram, possivelmente, pelo aumento da respiração do tecido vegetal e encurtamento do ciclo (Siqueira et al., 2001; Taiz \& Zeiger, 2004; Streck, 2005). No cultivo de outono, em todos os cenários com aumento de temperatura, o efeito benéfico do aumento da $\left[\mathrm{CO}_{2}\right]$ diminuiu (Figura $1 \mathrm{C}$ e D).

Tanto no cultivo de primavera quanto no de outono, as simulações com aumento simétrico nas temperaturas mínima e máxima diária do ar resultaram em menores valores de produtividade de tubérculos de batata, se comparados às simulações que consideraram aumentos assimétricos (Figura $1 \mathrm{~A}$ e C). Essa diferença entre os resultados das simulações nos cenários com aumentos simétricos e assimétricos ocorreu em função de penalização da fotossíntese que, no modelo matemático, decresce em valores de temperatura do ar acima de $25^{\circ} \mathrm{C}$, o que ocorreu principalmente nos cenários com maiores valores de temperatura máxima.

Conforme citado anteriormente, no modelo de Spitters (1987), o coeficiente utilizado para descrever a resposta da cultura ao aumento da concentração de $\mathrm{CO}_{2}$ é a EUR. Assim, foi considerado que as temperaturas diurnas do ar são mais limitantes na fotossíntese líquida e, portanto, as que mais interferem nos resultados das simulações. Como as temperaturas máximas são menos elevadas nos cenários de aumento assimétrico do que nos de aumento simétrico, a fotossíntese líquida é maior nos cenários $3 \mathrm{~b}, 4 \mathrm{~b}, 5 \mathrm{~b}, 6 \mathrm{~b}, 7 \mathrm{~b}$, e $8 \mathrm{~b}$, em razão da menor fotorrespiração do tecido vegetal. Um dos efeitos das altas temperaturas do ar em relação à temperatura ótima para a realização da fotossíntese, que é de $24^{\circ} \mathrm{C}$ para a cultura da batata (Timlin et al., 2006) em condições de clima subtropical, é acelerar a duração da fase de tuberização, o que resulta em menor produtividade, pois quanto menor a fase de 
tuberização, nas condições de cultivo do Brasil, menor a produtividade de tubérculos (Bisognin \& Streck, 2009).

No cultivo de primavera (Figura 1 A), nos cenários com aumentos simétricos e assimétricos nas temperaturas mínima e máxima diária do ar, a produtividade de tubérculos de batata começou a diminuir a partir do cenário com aumento de $3^{\circ} \mathrm{C}$ (cenários $5 \mathrm{a}$ e $5 \mathrm{~b}$ ). No entanto, no cultivo de outono, isso ocorreu já a partir do aumento de $1^{\circ} \mathrm{C}$ (cenários $3 \mathrm{a}$ e $3 \mathrm{~b}$, Figura $1 \mathrm{C}$ ). $\mathrm{Na}$ análise dos desvios de produtividade (Figuras 1 B e D), no cultivo na primavera, as reduções na produtividade de tubérculos de batata foram maiores do que no cultivo de outono, comparando-se ao cenário 1 .

No cultivo de primavera, nos cenários com aumento simétrico nas temperaturas mínima e máxima diária do ar, os desvios foram negativos, e indicaram redução de cerca de 7,33 e $58 \%$ na produtividade de tubérculos de batata, nos cenários com aumentos de 4,5 e $6^{\circ} \mathrm{C}$, respectivamente (Figura $1 \mathrm{~B}$ ), o que anulou o efeito benéfico do aumento da concentração de $\mathrm{CO}_{2}$ sobre a produtividade. Nos cenários com aumento assimétrico,
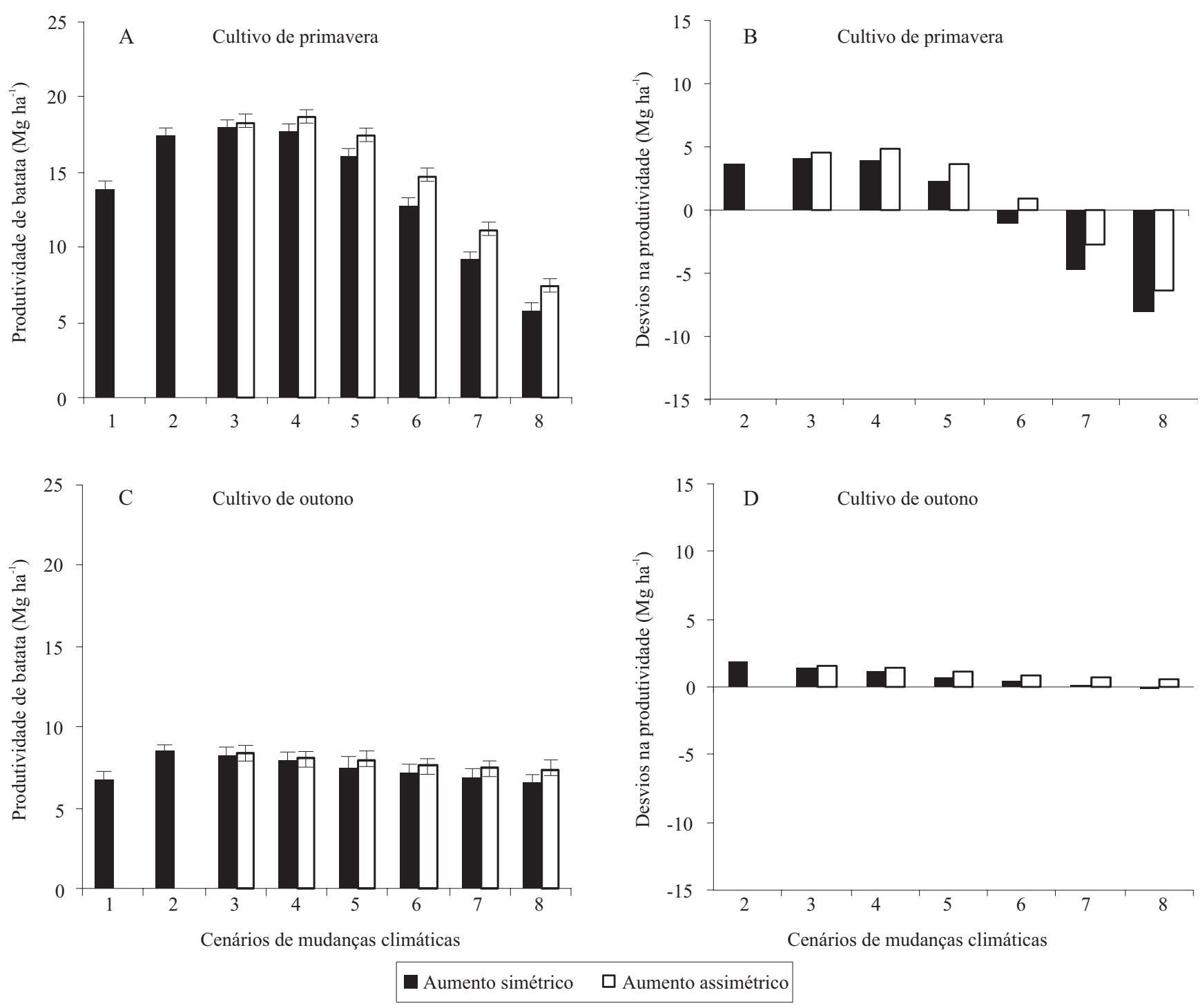

Figura 1. Produtividade e desvios da produtividade de tubérculos de batata em relação ao cenário 1 da Tabela 1 , para as datas de emergência 1/9 (A, B), no cultivo de primavera, e 22/2 (C, D) no cultivo de outono, em cenários de mudanças climáticas com aumentos simétricos e assimétricos nas temperaturas mínima e máxima diárias do ar em Santa Maria, RS. As barras de erro representam 1,0 desvio-padrão, da produtividade simulada em cada cenário. 
os desvios negativos corresponderam à redução na produtividade de tubérculos de batata em torno de 20 e $46 \%$, respectivamente, nos cenários com aumento de 5 e $6^{\circ} \mathrm{C}$, na temperatura média do ar.

No cultivo de outono, houve desvio negativo, com redução de $2 \%$ na produtividade de tubérculos de batata. Desse modo, o efeito benéfico do aumento da $\left[\mathrm{CO}_{2}\right]$ foi anulado somente no cenário com aumento simétrico de $6^{\circ} \mathrm{C}$, semelhantemente aos resultados reportados por Streck \& Alberto (2006a), para a cultura da soja, em Santa Maria, RS. Esses autores observaram desvios negativos somente para o cenário com aumento $6^{\circ} \mathrm{C}$. Nos demais cenários de aumentos simétricos e assimétricos nas temperaturas mínima e máxima, o efeito benéfico do aumento da $\left[\mathrm{CO}_{2}\right]$ não foi anulado pelo aumento da temperatura (Figura $1 \mathrm{D}$ ).

Segundo Hijmans (2003), resultados com simulações em diferentes locais do planeta indicam que a cultura da batata, assim como outras culturas, é bastante afetada pelo aumento na temperatura do ar. Estudos realizados em países de elevada latitude e clima temperado como, por exemplo, a Inglaterra (Davies et al., 1997), indicam um possível aumento na produtividade de tubérculos de batata diante das mudanças climáticas. Porém, em muitos países de latitudes baixas e médias, o que inclui o Brasil, o efeito das elevadas temperaturas do ar poderá ocasionar a diminuição na produtividade de tubérculos de batata, e uma estratégia para minimizar o efeito negativo das altas temperaturas nestes locais será alterar as datas de plantio (Hijmans, 2003). Esta hipótese foi testada em Santa Maria (Figuras 2 e 3) e mostrou que, tanto no cultivo de primavera quanto no de outono, as produtividades simuladas nos cenários com aumentos simétricos e assimétricos nas temperaturas mínima e máxima do ar apresentaram a mesma tendência de aumento, quando as datas de emergência foram adiantadas ou atrasadas em relação às datas de emergência da primavera, 1/9, e de outono, 22/2, analisadas na Figura 1.

Nas datas de emergência antecipadas de 18/8 (Figura $2 \mathrm{~A}$ e B) e 25/8 (Figura $2 \mathrm{C}$ e D), para o cultivo de primavera da cultura da batata, a produtividade simulada, na média de todos os cenários e nos cenários mais quentes, em que se verificam aumentos acima de $2^{\circ} \mathrm{C}$, foi superior ao cultivo com data de emergência de 1/9 (Figura 1 A e B), enquanto nas datas de emergência posteriores de 8/9 (Figura 2 E e F) e $15 / 9$ (Figura $2 \mathrm{G} \mathrm{e} \mathrm{H}$ ), se comparados principalmente aos cenários $8 \mathrm{a}$ e $8 \mathrm{~b}$, que apresentaram os maiores aumentos na temperatura, a produtividade simulada foi muito afetada.

Os desvios na produtividade foram menores nas duas datas de emergência antecipadas, se comparados aos desvios na data de 1/9. Na data de emergência 18/8, ocorreu desvio negativo somente para o cenário $8 \mathrm{a}$ e $8 \mathrm{~b}$, ou seja, somente diante do aumento de $6^{\circ} \mathrm{C}$ na temperatura o efeito benéfico do aumento de $\mathrm{CO}_{2}$ seria anulado (Figura $2 \mathrm{~B}$ ). O desvio negativo nesse cenário provocou redução de $26 \%$ na produtividade de tubérculos de batata, no cenário com aumento simétrico nas temperaturas, e de 7\%, no cenário com aumento assimétrico nas temperaturas. $\mathrm{Na}$ data de emergência 25/8 (Figura $2 \mathrm{D}$ ), o efeito benéfico do aumento da $\left[\mathrm{CO}_{2}\right]$ foi anulado pelo aumento de 5 e $6^{\circ} \mathrm{C}$ na temperatura, com redução na produtividade de 16 e $43 \%$, em cenários de aumento simétrico na temperatura do ar, e de $27 \%$ com o aumento de $6^{\circ} \mathrm{C}$ na temperatura, no cenário assimétrico. Nas duas datas de emergência tardias (8/9 e 15/9), o efeito benéfico do aumento de $\mathrm{CO}_{2}$ foi anulado a partir do aumento de $3^{\circ} \mathrm{C}$ (Figura 2 $\mathrm{F} \mathrm{e} \mathrm{H).}$

Nas datas de emergência antecipadas de 8/2 (Figura 3 A e B) e 15/2 (Figura 3 C e D), para o cultivo de outono, as produtividades simuladas foram semelhantes à produtividade observada com a emergência em 22/2 (Figura $1 \mathrm{C}$ e D), quando consideradas as médias de todos os cenários. Os desvios na produtividade nas duas datas de emergência antecipadas, assim como na época recomendada (Figura 1), mostram que o efeito do aumento da $\left[\mathrm{CO}_{2}\right]$ praticamente não é anulado pelo aumento da temperatura do ar. Os desvios são negativos somente nos cenários com aumentos simétricos de 5 e $6^{\circ} \mathrm{C}$ na temperatura mínima e máxima diária do ar (Figura 1 D, 3 B e D). Na data de emergência de $8 / 2$, em que a temperatura do ar durante a estação de crescimento é mais elevada, ocorreram maiores valores de desvios negativos na produtividade (Figura $3 \mathrm{~B}$ ). $\mathrm{O}$ efeito da $\left[\mathrm{CO}_{2}\right]$ na data de emergência $8 / 2$ foi anulado com aumentos de 5 e $6^{\circ} \mathrm{C}$, nos cenários de aumento simétrico, o que representou uma redução na produtividade de 5 e 9\%, respectivamente (Figura 3 B). Na data de emergência 22/2, o efeito das altas temperaturas foi menor (Figura 1 D), o que ocasionou redução na produtividade de apenas $2 \%$, diante do aumento de $6^{\circ} \mathrm{C}$ na temperatura, no cenário com aumento simétrico. Quanto ao atraso na data 

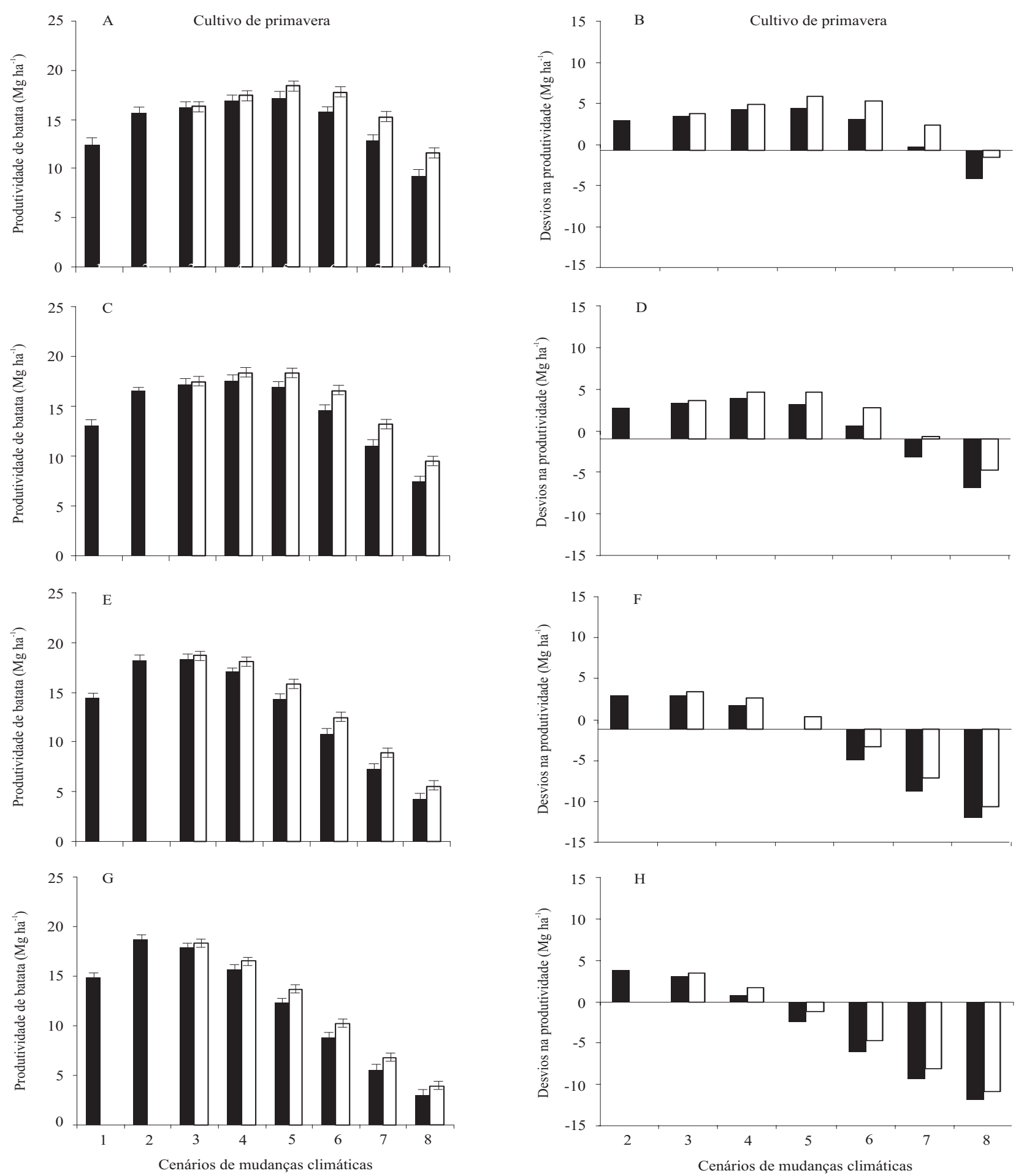

- Aumento simétrico $\square$ Aumento assimétrico

Figura 2. Produtividade e desvios da produtividade de tubérculos de batata em datas de emergência anteriores - 18/8 e 25/8 $(\mathrm{A}, \mathrm{B}, \mathrm{C}, \mathrm{D})$ - e posteriores - 8/9 e 15/9 (E, F, G, H) - à data de emergência, 1/9, utilizada na Figura $1 \mathrm{~A}$ e $\mathrm{B}$, no cultivo de primavera, em cenários de mudanças climáticas com aumentos simétricos e assimétricos nas temperaturas mínima e máxima diárias do ar, em Santa Maria, RS. As barras de erro representam um desvio-padrão da produtividade simulada em cada cenário. 

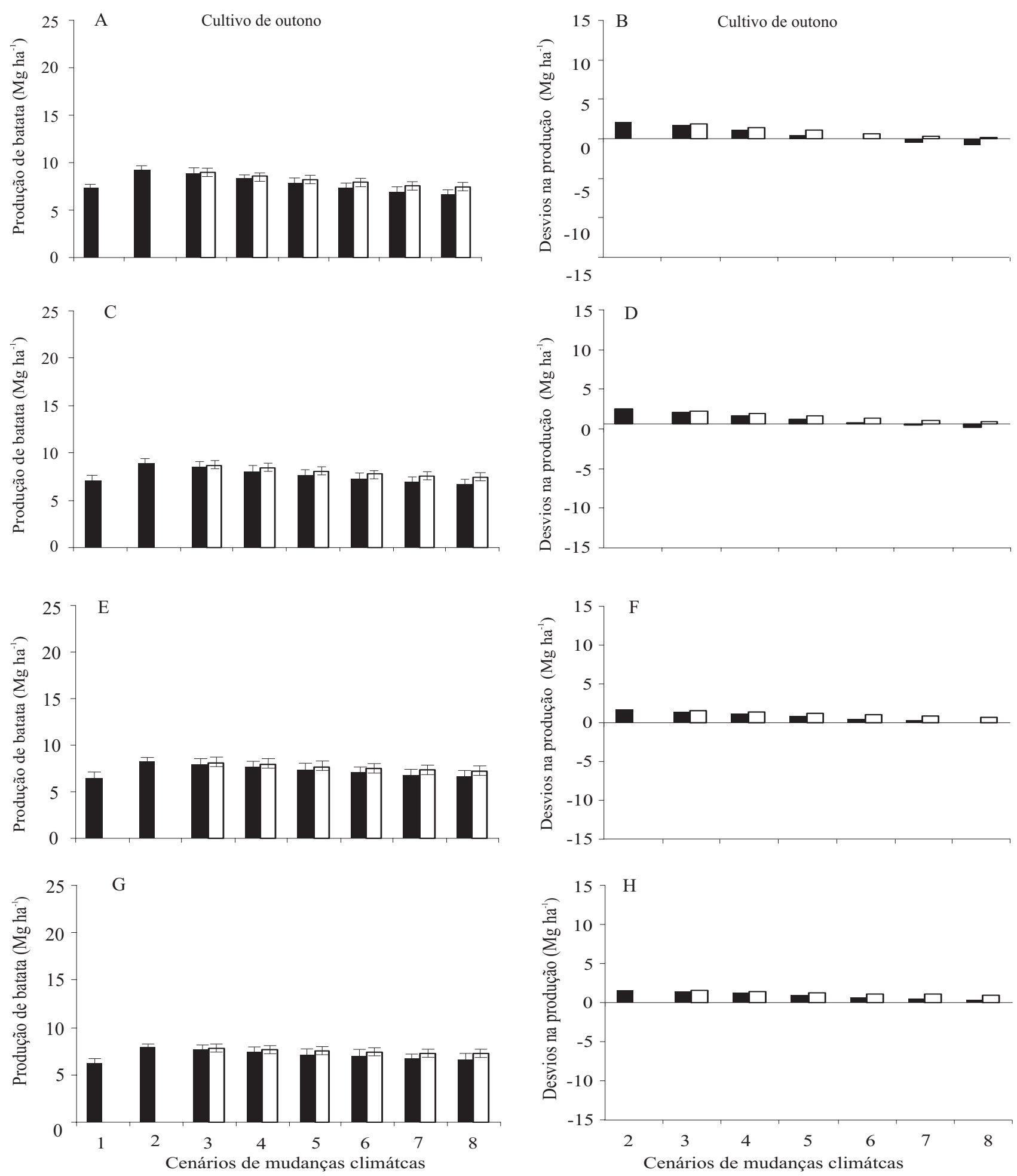

Aumento simétrico $\square$ Aumento assimétrico

Figura 3. Produtividade e desvios da produtividade de tubérculos de batata em datas de emergência anteriores - $8 / 2$ e $15 / 2$ $(\mathrm{A}, \mathrm{B}, \mathrm{C}, \mathrm{D})$ - e posteriores - 1/3 e $8 / 3(\mathrm{E}, \mathrm{F}, \mathrm{G}, \mathrm{H})$ - à data de emergência 22/02, usada na Figura $1 \mathrm{C}$ e $\mathrm{D}$, no cultivo de outono, em cenários de mudanças climáticas com aumentos simétricos e assimétricos nas temperaturas mínima e máxima diárias do ar, em Santa Maria, RS. As barras de erro representam um desvio padrão da produtividade simulada em cada cenário. 
de emergência, no cultivo de outono, foi estimado aumento na produtividade de tubérculos de batata, ou seja, ocorreram somente desvios positivos (Figura $3 \mathrm{~F} \mathrm{e}$ $\mathrm{H})$, o que indica que o efeito do aumento da $\left[\mathrm{CO}_{2}\right]$ não foi anulado pelos aumentos nas temperaturas mínima e máxima diárias do ar. Streck et al. (2006) simularam aumento na duração da fase emergência-colheita, com o aumento da temperatura no cultivo de outono, o que ajuda a explicar os desvios positivos observados nas datas mais tardias (Figura $3 \mathrm{~F}$ e $\mathrm{H}$ ), já que a cultura permanece mais tempo na fase de enchimento de tubérculos.

Diante das mudanças observadas na produtividade de tubérculos de batata decorrentes de aumentos da temperatura do ar, pode-se inferir que a estratégia de antecipar a data de plantio no cultivo de primavera e atrasar no cultivo de outono é válida na região de Santa Maria, RS.

\section{Conclusões}

1. No cultivo de primavera, o aumento simétrico na temperatura de $4^{\circ} \mathrm{C}$ e assimétrico de $5^{\circ} \mathrm{C}$ é suficiente para anular o efeito benéfico do aumento da concentração de dióxido de carbono sobre a produtividade de tubérculos de batata.

2. No cultivo de outono, o aumento da temperatura praticamente não afeta a produtividade de tubérculos de batata.

3. A antecipação na data de plantio no cultivo de primavera e o atraso no cultivo de outono diminuem o impacto negativo do aumento da temperatura do ar sobre a produtividade de tubérculos de batata em Santa Maria, RS.

\section{Agradecimentos}

Ao Conselho Nacional de Desenvolvimento Científico e Tecnológico e à Coordenação de Aperfeiçoamento de Pessoal de Nível Superior, pela concessão de bolsas de pesquisa aos autores.

\section{Referências}

ANDRESEN, J.A.; ALAGARSWAMY, G.; ROTZ, C.A.; RITCHIE, J.T.; LEBARON, A.W. Weather impacts on maize, soybean, and alfalfa production in the Great Lakes region, 1895-1996. Agronomy Journal, v.93, p.1059-1070, 2001.

ASSAD, E.D.; PINTO, H.S.; ZULLO JUNIOR, J.; ÁVILA, A.M.H. Impacto das mudanças climáticas no zoneamento agroclimático do café no Brasil. Pesquisa Agropecuária Brasileira, v.39, p.1057-1064, 2004.

BISOGNIN, D.A.; MÜLLER, D.R.; STRECK, N.A.; ANDRIOLO, J.L.; SAUSEN, D. Desenvolvimento e rendimento de clones de batata na primavera e no outono. Pesquisa Agropecuária Brasileira, v.43, p.699-705, 2008.

BISOGNIN, D.A.; STRECK, N.A. Desenvolvimento e manejo das plantas para alta produtividade e qualidade da batata. Itapetininga: Associação Brasileira da Batata, 2009. 30p.

DAVIES, A.; JENKINS, T.; PIKE, A.; SHAO, J.; CARSON, I.; POLLOCK, C.J.; PARRY, M.L. Modelling the predicted geographic and economic response of UK cropping systems to climate change scenarios: the case of potatoes. Annals of Applied Biology, v.130, p.167-178, 1997.

GONDIM, R.S.; CASTRO, M.A.H. de; EVANGELISTA, S.R. de M.; TEIXEIRA, A. dos S.; FUCK JÚNIOR, S.C. de F. Mudanças climáticas e impactos na necessidade hídrica das culturas perenes na Bacia do Jaguaribe, no Estado do Ceará. Pesquisa Agropecuária Brasileira, v.43, p.1657-1664, 2008.

HIJMANS, R.J. The Effect of climate change on global potato production. American Journal of Potato Research, v.80, p.271-279, 2003.

INTERGOVERNMENTAL PANEL ON CLIMATE CHANGE. Climate change 2007: the physical science basis: contribution of Working Group I to the Fourth Assessment Report of the Intergovernmental Panel on Climate Change. Cambridge: IPCC, 2007. 989p.

KARL, T.R.; KUKLA, G.; RAZUVAYEV, V.N.; CHANGERY, M.J.; QUAYLE, R.G.; HEIM JUNIOR, R.R.; EASTERLING, D.R.; BIN FU, C. Global warming: evidence for asymmetric diurnal temperature change. Geophysical Research Letters, v.18, p.2253-2256, 1991.

KERR, R.A. Millennium's hottest decade retains its title, for now. Science, v.307, p.828-829, 2005.

KOOMAN, P.L.; HAVERKORT, A.J. Modelling development and growth of the potato crop influenced by temperature and daylength: LINTUL-POTATO. In: HAVERKORT, A.J.; MACKERRON, D.K.L. Potato ecology and modeling of crops under conditions of limiting growth. Dordrecht: Kluwer Academic, 1995. v.3, p.41-60.

LAGO, I.; STRECK, N.A.; ALBERTO, C.M.; OLIVEIRA, F.B.; DE PAULA, G.M. Impact of increasing mean air temperature on the development of rice and red rice. Pesquisa Agropecuária Brasileira, v.43, p.1441-1448, 2008.

NYENDE, A.B.; SCHITTENHELM, S.; MIX-WAGNER, G.; GREEF, J.M. Yield and canopy development of field grown potato plants derived from synthetic seeds. European Journal of Agronomy, v.22, p.175-184, 2005.

RICHTER, G.M.; SEMENOV, M.A. Modelling impacts of climate change on wheat yields in England and Wales: assessing drought risks. Agricultural Systems, v.84, p.77-97, 2005.

ROSENZWEIG, C.J.; PHILLIPS, J.; GOLDBERG, R.; CARROLL, J.; HODGES, T. Potential impacts of climate change on citrus and potato production in the US. Agricultural Systems, v.52, p.455-479, 1996. 
SCHAPENDONK, A.H.C.M.; POT, C.S.; GOUDRIAAN, J. Simulated effects of elevated carbon dioxide concentration and temperature on the productivity of potato. In: HAVERKORT, A.J.; MACKERRON, D.K.L. Potato ecology and modeling of crops under conditions of limiting growth. Dordrecht: Kluwer Academic, 1995. v.3, p.101-114.

SEMENOV, M.A.; BROOKS, R.J.; BARROW, E.M.; RICHARDSON, C.W. Comparison of the WGEN and LARS-WG stochastic weather generators for diverse climates. Climate Research, v.10, p.95-107, 1998.

SIQUEIRA, O.J.W. de; STEINMETZ, S.; FERREIRA, M.F.; COSTA, A.C.; WOZNIAK, M.A. Mudanças climáticas projetadas através dos modelos GISS e reflexos na produção agrícola brasileira. Revista Brasileira de Agrometeorologia, v.8, p.311-320, 2000.

SIQUEIRA, O.J.W. de; STEINMETZ, S.; SALLES, L.A.B. Efeitos potenciais das mudanças climáticas na agricultura brasileira e estratégias adaptativas para algumas culturas. In: LIMA, M.A. de; CABRAL, O.M.R.; GONZALES MIGUEZ, J.D. Mudanças climáticas globais e a agropecuária brasileira. Jaguariúna: Embrapa Meio Norte, 2001. p.33-63.

SPITTERS, C.J.T. An analysis of variation in yield among potato cultivars in terms of light absorption, light utilization and dry matter partitioning. Acta Horticulturae, v.214, p.71-84, 1987.

STRECK, N.A. Climate change and agroecosystems: the effect of elevated atmospheric $\mathrm{CO}_{2}$ and temperature on crop growth, development, and yield. Ciência Rural, v.35, p.730-740, 2005.
STRECK, N.A.; ALBERTO, C.M. Estudo numérico do impacto da mudança climática sobre o rendimento de trigo, soja e milho. Pesquisa Agropecuária Brasileira, v.41, p.1351-1359, 2006 a.

STRECK, N.A.; ALBERTO, C.M. Simulação do impacto da mudança climática sobre a água disponível do solo em agroecossistemas de trigo, soja e milho em Santa Maria, RS. Ciência Rural, v.36, p.424-433, 2006 b.

STRECK, N.A.; LAGO, I.; ALBERTO, C.M.; BISOGNIN, D.A. Simulação do desenvolvimento de batata cultivar Asterix em cinco cenários de mudança climática em Santa Maria, RS. Bragantia, v.65, p.693-702, 2006.

TAIZ, L.; ZEIGER, E. Fisiologia vegetal. 3.ed. Porto Alegre: Artemed, 2004. 719p.

TIMLIN, D.; RAHMAN, S.M.L.; BAKER, J.; REDDY, V.R.; FLEISHER, D.; QUEBEDEAUX, B. Whole plant photosynthesis, development, and carbon partitioning in potato as a function of temperature. Agronomy Journal, v.98, p.1195-1203, 2006.

TUBIELLO, F.N.; DANATELLI, M.; ROSENZWEIG, C.; STOCKLE, C.O. Effects of climate change and elevated $\mathrm{CO}_{2}$ on cropping systems: model predictions at two Italian locations. European Journal of Agronomy, v.13, p.179-189, 2000.

WEISS, A.; HAYS, C.J.; WON, J. Assessing winter wheat response to climate change scenarios: a simulation study in the U.S. great plains. Climatic Change, v.58, p.119-147, 2003.

Recebido em 20 de janeiro de 2010 e aprovado em 12 de março de 2010 